\title{
Corrigendum of \\ A Note on the Exceptional Set for Goldbach's Problem in Short Intervals
}

Mh. Math. 116, 275-282 (1993)

By

J. Kaczorowski, A. Perelli, and J. Pintz

Professor D. A. Goldston pointed out that there is a gap in Eq. (18) and proposed the following elegant way to correct it. We note that our original argument would in fact lead to a result with an additional factor $\log H$ in the first estimate of the Theorem and an analogous modification of its Corollary, while Goldston's argument shows that both, the Theorem and the Corollary hold has they are.

The first six lines of Sect. 3, p. 281, should be replaced by the following.

Let $t(k)=1 / H \max (2 H-|k|, 0)$. Due to our choice of $Q$, writing \|\|$=$ distance from the nearest integer and using the properties of the Fejer kernel,

$$
K(\alpha)=\sum_{k=-\infty}^{\infty} t(k) e(-k \alpha) \ll 1 / H \min \left(H^{2}, \frac{1}{\|\alpha\|^{2}}\right),
$$

we obtain that

$$
\begin{aligned}
& \sum_{N \leqslant n \leqslant N+H}\left|\int_{m} S(\alpha)^{2} e(-n \alpha) \mathrm{d} \alpha\right|^{2} \leqslant \sum_{n} t(n-N)\left|\int_{m} S(\alpha)^{2} e(-n \alpha) \mathrm{d} \alpha\right|^{2}= \\
& =\sum_{n} t(n-N) \iint_{m m} \overline{S(\xi)^{2}} S(\alpha)^{2} e(-n(\alpha-\xi)) \mathrm{d} \alpha \mathrm{d} \xi \ll \\
& \ll N L \max _{\xi \in[0,1]}|S(\alpha)|^{2}|K(\alpha-\xi)| \mathrm{d} \alpha \ll
\end{aligned}
$$


$\ll N L \max _{\xi \in[0,1]} \sum_{k=-2 H}^{2 H} \frac{H}{k^{2}+1} \int_{(\xi-k / 2 H-1 / H, \xi-k / 2 H+1 / H) \cap m}|S(\alpha)|^{2} \mathrm{~d} \alpha \ll$ $\ll H N L \max _{\xi \in[0,1]} \int_{(\xi-1 / H, \xi+1 / H) \cap m}|S(\alpha)|^{2} \mathrm{~d} \alpha \ll$

$$
\ll H N L \max _{\substack{p<q \leqslant Q \\(a, q)=1}} \int_{-1 / q Q}^{1 / q Q}\left|S\left(\frac{q}{q}+\eta\right)\right|^{2} \mathrm{~d} \eta .
$$

We wish to thank Prof. Goldston for providing us the above argument.

\section{JERZY KACZOROWSKI}

Institute of Mathematics

A. Mickiewicz University

Poznań, Poland

\section{JANOS PINTZ}

Mathematical Institute of the Hungarian Academy of Sciences

Reáltanoda u. 13-15

H-1364 Budapest

Hungary

\author{
Alberto Perelli \\ Dipartimento di Matematica \\ Via L. B. Alberti 4 \\ 16132 Geneva \\ Italy \\ e-mail perelli@ dima. unige.it
}

